\title{
Screening for Anti-diabetic Effects of Prescribed Korean Traditional Medicines
}

\author{
Seon Mi Shin ${ }^{1 \dagger}$, Yong Joon Jeong ${ }^{2 \dagger}$, Dae Won Park ${ }^{2}$, Heung Ko ${ }^{1}$, Gi tae Kim ${ }^{1}$, Ee-Hwa Kim ${ }^{3 *}$, \\ Taeseong Kim ${ }^{4}$, Eun-Hwa Sohn ${ }^{4}$, Jung-Eun Kwon ${ }^{2}$, Hyun Jung Koo ${ }^{2}$ and Se Chan Kang ${ }^{2}$ * \\ ${ }^{1}$ Department of Oriental Internal Medicine, Semyung University, Jecheon 390-711, Korea \\ ${ }^{2}$ Department of Life Science, Gachon University, Seongnam 461-701, Korea \\ ${ }^{3}$ Department of Meridian \& Acupoint, Semyung University, Jecheon 390-711, Korea \\ ${ }^{4}$ Department of Herbal Medicine Resource, Kangwon National University, Samcheok 245-711, Korea
}

\begin{abstract}
Herb extracts commercially used in Korea were screened for PPAR- $\gamma$ agonist test and a-glucosidase inhibition assay. Total 16 herb plants had a PPAR- $\gamma$ agonist activity. Specially, Alisma orientale Juz (108.41\%), Ephedra sinica (98.22\%), Sasa japonica Makino var. purpurascens Nakai (140.68\%), Astragalus membranaceus Bunge (106.79\%) and Cnidium officinale Makino (113.00\%) showed high PPAR- $\gamma$ agonist activity rate compared with rosiglitazone's (167.46\%). And Cornus officinalis S. et Z. (90.3\%), Cinnamomum cassia Blume (89.2\%), Psoralea corylifolia L. (89.8\%), Paeonia japonica (Makino) Miyabe (92.4\%) and Paeonia suffruticosa Andr (93.2\%), showed high a-glucosidase inhibition rates. These results support previous reports of the efficacy of Oriental medicinal plants used for diabetes mellitus.
\end{abstract}

Key words - Korean traditional medicine, Screening, PPAR- $\gamma$ agonist, a-Glucosidase inhibition, Diabetes mellitus

\section{Introduction}

The incidence of type II diabetes mellitus (T2DM) has reached epidemic proportions in western and developing countries, with an estimated 194 million people afflicted. Peripheral insulin resistance is a key feature of T2DM and results from a combination of sedentary lifestyle, unhealthy dietary habits, and genetic predisposition (Skyler, 2004). Insulin resistance is also implicated in a number of life threatening disorders collectively referred to as the metabolic syndrome (Hansen, 1999). T2DM affects many metabolic pathways in different tissues, many of which are potential targets for drug treatment (van de Venter et al., 2008). Type II diabetes is characterized by hepatic and peripheral (muscle and adipose tissue) insulin resistance. The pancreas compensates by secreting more insulin, but eventually the beta cells will fail to sustain this (Cerasi, 2000), at which stage the patient requires insulin treatment. During the stage when insulin is

\section{${ }^{*}$ Corresponding author.}

E-mail : sckang73@gachon.ac.kr / kimeh@semyung.ac.kr ${ }^{\dagger}$ Seon Mi Shin and Yong Joon Jeong contributed equally. still produced, various other classes of drugs, in combination with lifestyle alterations, can be used to manage the disease (Matthaei et al., 2000). These drugs act through a number of different ways or targets to reduce blood glucose levels. When screening for an anti-diabetic agent, it is important to include as many of these targets as possible to ensure that possible 'hits' are not excluded (van de Venter et al., 2008).

PPARs (peroxisome proliferator-activated receptors), the ligand-activated nuclear transcription factors, regulate the gene expression of adipocyte differentiation, lipogenesis, and glucose metabolism, and are involved in type 2 diabetes, dyslipidemia, atherosclerosis, obesity, and other metabolic diseases (Evans et al., 2004; Pakala et al., 2004). Ligands of PPARs have been used for treating these diseases clinically for decades (Huang et al., 2006). The thiazolidinedione class of insulin-sensitizing compounds such as rosiglitazone and pioglitazone, which lower hyperglycemia, is PPAR- $\gamma$ agonists (Stales and Fruchart, 2005). However, the limitations and the side-effects of these drugs have driven researchers to look for a new type of drug that could be of benefit for the treatment of some of these metabolic disorders.

3T3-L1 fibroblasts transformed to adipocyte when differenti- 
ation inducer like insulin, dexamethasone and IBMX (3-Isobutyl1-methylxanthine) are added (Gerhold et al., 2002), but this mechanism has not been known clearly yet. Known mechanisms is that adipocyte differentiation is starting to be expressed when the transcription factors which operate in genes at fatty tissues specifically is expressed. This transcription factors are peroxisome proliferator-activated receptor $\gamma(\operatorname{PPAR}-\gamma), \mathrm{CCAAT} /$ enhancing binding protein (C/EBP), adipocyte differentiation and determinator factor 1 and sterol regulatory element binding protein 1c. It is known that substance induced differentiation can convert fibroblast to adipocyte by promoting expressing of these transcription factors. Especially PPAR- $\gamma$ is able to control the cell growth, start converting of adipocyte, and regulate the adipocyte differentiation. Also C/EBP is expressed in adipocyte and C/EBP helps the PPAR- $\gamma$ convert preadipocyte to adipocyte (Yamamoto et al., 2002). Therefore, it is considered that the herb plants, which can differentiate 3T3-L1 fibroblast and increase the lipid accumulation, are able to act as a PPAR- $\gamma$ agonist.

Current medication options for the treatment of diabetes are relatively limited, have non-negligible side effects, and must often be prescribed in combination (Cheng and Fantus, 2005). The use of natural health products as complementary or alternative approaches to existing medications is growing in popularity. While these have been the object of very few rigorous scientific studies, it is clear that many plants possess hypoglycemic activity, some having considerable anti-diabetic potential (Marles and Farnsworth, 1995). The prevalence of use of complementary and alternative medicine (CAM) is high among people with diabetes (Wolsko et al., 2002) and those seeking to prevent chronic diseases that include diabetes (Harnack et al., 2001).

Active ingredient of medicinal plants that make Korea herb medicine is secondary metabolites with bioactive substances. Recently insulin like substances from medicinal plants as secondary metabolites, attention has been separated. It has been reported that royal jelly effects on the prevention and treatment of diabetes, because trans-10-hydroxy-2-decanoic acid in royal jelly is insulin like substance (Kameda et al., 1996). Also, It has been reported that bakuchiol from Ortholobium pubescens (which is a traditional medicinal plants in Peru) is insulin material (Krenisky et al., 1999).

In Korea, a traditional medicine, diabetes mellitus could be divided in "So-gal", "So-joong" and "So-sin" broadly and it has been evaluated that So-gal part in Donguibogam is described in detail as these day's diabetes mellitus, and it is amazing. Also, in Asia including Korea, various herbs have been used for treatment of diabetes mellitus. Some studies had found out the anti-diabetic effects of theses herbs, but there are not enough evidences of their anti-diabetic effects.

In this study, we screened the frequent medicinal herbs in Korea traditional medical book, "Donguibogam" by PPAR- $\gamma$ agonist test and a-glucosidase inhibition assay.

\section{Materials and Methods}

\section{Literature search}

We had researched the Korea traditional medical book, "Donguibogam", and found out the prescription (Table 1) and frequent herbs (Table 2). There were total 54 Korea

Table 1. Korean traditional herb medicines used for treating diabetes in Donguibogam

\begin{tabular}{ll}
\hline \hline \multicolumn{1}{c}{ Herbmed } & \multicolumn{1}{c}{ Prescription } \\
\hline Backhogainsamtang & $\begin{array}{l}\text { Gypsum, Anemarrhena asphodeloides Bunge, Glycyrrhiza uralensis Fisch, Oryza sativa L., Panax } \\
\text { ginseng C. A. Meyer }\end{array}$ \\
Bosinjihwangwon & $\begin{array}{l}\text { Phellodendron amurense Rupr., Rehmannia glutinosa Libosch., Poria cocos Wolf, Rehmannia } \\
\text { glutinosa } \text { Libosch., Asparagus cochinchinensis Merr., Panax ginseng C. A. Meyer, Chrysanthemum } \\
\text { indicum L., Scutellaria baicalensis Georgi, Angelica gigas Nakai, Citrus aurantium L., Liriope } \\
\text { platyphylla } \text { Wang. et Tang, Scutellaria baicalensis Georgi }\end{array}$ \\
& Nelumbo nucifera Gaertn, Poria cocos Wolf, Panax ginseng C. A. Meyer, Astragalus membranaceus \\
Cheongsimyeonjaeum & Lycium chinense Mill., Glycyrrhiza uralensis Fisch \\
\hline
\end{tabular}


Korean J. Plant Res. 25(6) : 670 681 (2012)

Table 1. Continued

\begin{tabular}{cl}
\hline \hline Herbmed & \multicolumn{1}{c}{ Prescription } \\
\hline Cheongsinbogitang & Cimicifuga heracleifolia Kom., Bupleurum falcatum L., Angelica gigas Nakai, Schizonepeta tenuifolia \\
& Briq, Sinomenium acutum Rehder et E. H. Wilson, Prunus persica (L.) Batsch, Phellodendron \\
& amurense Rupr., Coptis japonica Makino, Anemarrhena asphodeloides Bunge, Glycyrrhiza uralensis \\
& Fisch, Gypsum, Rehmannia glutinosa Libosch., Asarum heterotropoides F. Maekawa, Prunus \\
& armeniaca L. var. ansu Maxim, Zanthoxylum bungeanum, Carthamus tinctorius L.
\end{tabular}

Cheonwhasan

Dahwanggamchoeumja

Eegwonsan

Gagambackchulsan

Gagambackhotang

Gagamsingihwan

Gamijeonssibackchoolsan

Gangsimtang

Goryuntang

Hwahyulikgitang

Hyuntodan

Insambockreyngsan
Trichosanthes kirilowii Maxim, Rehmannia glutinosa Libosch., Pueraria lobata (Willd.) Ohwi, Liriope platyphylla Wang. et Tang, Schizandra chinensis Bailon, Glycyrrhiza uralensis Fisch

Rheum undulatum L., Glycyrrhiza uralensis Fisch, Glycine semen

Talcum, Glycyrrhiza uralensis Fisch

Pueraria lobata (Willd.) Ohwi, Panax ginseng C. A. Meyer, Atractylodes japonika Koidz., Poria cocos Wolf, Aucklandia lappa Decne., Anemarrhena asphodeloides Bunge, Phellodendron amurense Rupr., Glycyrrhiza uralensis Fisch, Schizandra chinensis Bailon

Gypsum, Anemarrhena asphodeloides Bunge, Panax ginseng C. A. Meyer, Phellodendron amurense Rupr., Scrophularia buergeriana Miq., Glycyrrhiza uralensis Fisch, Schizandra chinensis Bailon, Oryza sativa L.

Rehmannia glutinosa Libosch., Paeonia suffruticosa Andr, Poria cocos Wolf, Cornus officinalis S. et Z., Schizandra chinensis Bailon, Alisma orientale Juz., Cervus nippon hortulorum, Dioscorea batatas Decne., Cinnamomum cassia Blume, Aquilaria agallocha Roxb.

Pueraria lobata (Willd.) Ohwi, Panax ginseng C. A. Meyer, Atractylodes japonika Koidz., Poria cocos Wolf, Agastache rugosa (Fisc.r et Mey.) Kuntze, Glycyrrhiza uralensis Fisch, Aucklandia lappa Decne., Bupleurum falcatum L., Citrus aurantium L., Schizandra chinensis Bailon

Trichosanthes kirilowii Maxim, Panax ginseng C. A. Meyer, Polygala tenuifolia Willd, Angelica gigas Nakai, Rehmannia glutinosa Libosch., Poria cocos Wolf, Astragalus membranaceus Bunge,

Schizandra chinensis Bailon, Glycyrrhiza uralensis Fisch, Zizyphus jujuba var. inermis

Melia azedarach, Moschus moschiferus

Phellodendron amurense Rupr., Cimicifuga heracleifolia Kom., Rehmannia glutinosa Libosch., Coptis japonica Makino, Gypsum, Prunus armeniaca L. var. ansu Maxim, Prunus persica (L.) Batsch, Anemarrhena asphodeloides Bunge, Sinomenium acutum Rehder et E. H. Wilson, Ostericum koreana Kitagawa, Angelica gigas Nakai, Bupleurum falcatum L., Ephedra sinica, Glycyrrhiza uralensis Fisch, Carthamus tinctorius L.

Cuscuta chinensis Lamark, Schizandra chinensis Bailon, Poria cocos Wolf, Nelumbo nucifera Gaertn, Dioscorea batatas Decne.

Talcum, Gypsum rubrum, Glycyrrhiza uralensis Fisch, Poria cocos Wolf, Pueraria lobata (Willd.) Ohwi, Scutellaria baicalensis Georgi, Mentha arvensis var. piperascens MALINV, Rheum undulatum L., Forsythia viridissima Lindley, Panax ginseng C. A. Meyer, Atractylodes japonika Koidz., Alisma orientale Juz., Platycodon grandiflorum A. DC, Gardenia jasminoides Ellis, Trichosanthes kirilowii Maxim, Amomum villosum Lour. 
Table 1. Continued

\begin{tabular}{|c|c|}
\hline Herbmed & Prescription \\
\hline Insamseokgotang & Gypsum, Anemarrhena asphodeloides Bunge, Panax ginseng C. A. Meyer, Glycyrrhiza uralensis Fisch \\
\hline Jaeumyangyoungtang & $\begin{array}{l}\text { Angelica gigas Nakai, Panax ginseng C. A. Meyer, Rehmannia glutinosa Libosch., Liriope platyphylla } \\
\text { Wang. et Tang, Paeonia japonica (Makino) Miyabe, Anemarrhena asphodeloides Bunge, } \\
\text { Phellodendron amurense Rupr., Glycyrrhiza uralensis Fisch, Schizandra chinensis Bailon }\end{array}$ \\
\hline Joosahwangreonwon & Coptis japonica Makino, Rehmannia glutinosa Libosch., Cinnabaris \\
\hline Macmoondongeumja & $\begin{array}{l}\text { Liriope platyphylla Wang. et Tang, Anemarrhena asphodeloides Bunge, Trichosanthes kirilowii } \\
\text { Maxim, Panax ginseng C. A. Meyer, Schizandra chinensis Bailon, Pueraria lobata (Willd.) Ohwi, } \\
\text { Poria cocos Wolf, Rehmannia glutinosa Libosch., Glycyrrhiza uralensis Fisch, Sasa japonica Makino } \\
\text { var. purpurascens Nakai }\end{array}$ \\
\hline Meahwatang & Oraza sativa L. var. glutinosa, Matsumura, Morus alba Linne \\
\hline Moondongeumja & $\begin{array}{l}\text { Liriope platyphylla Wang. et Tang, Schizandra chinensis Bailon, Panax ginseng C. A. Meyer, Lycium } \\
\text { chinense Mill., Poria cocos Wolf, Glycyrrhiza uralensis Fisch }\end{array}$ \\
\hline Nahaeumja & $\begin{array}{l}\text { Gypsum, Anemarrhena asphodeloides Bunge, Glycyrrhiza uralensis Fisch, Ledebouriella seseloides } \\
\text { (Hoffm.) Wolff, Panax ginseng C. A. Meyer., Forsythia viridissima Lindley, Amomum cardamomum } \\
\text { L., Platycodon grandiflorum A. DC, Cimicifuga heracleifolia Kom., Pinellia ternata Breitenbach, } \\
\text { Zingiber officinale Roscoe }\end{array}$ \\
\hline
\end{tabular}

Nockyounghwan

Liriope platyphylla Wang. et Tang, Cervus nippon hortulorum, Rehmannia glutinosa Libosch., Astragalus membranaceus Bunge, Schizandra chinensis Bailon. Gallus gallus var. domesticus, Cistanche deserticola Y.C. Ma, Cornus officinalis S. et Z., Psoralea corylifolia L., Achyranthes bidentata Blume, Panax ginseng C. A. Meyer, Poria cocos Wolf, Lycium chinense Mill., Scrophularia buergeriana Miq.

Ockcheonsan

Trichosanthes kirilowii Maxim, Pueraria lobata (Willd.) Ohwi, Liriope platyphylla Wang. et Tang, Rehmannia glutinosa Libosch., Schizandra chinensis Bailon, Glycyrrhiza uralensis Fisch, Oraza sativa L. var. glutinosa Matsumura

Ockcheonwhan

Trichosanthes kirilowii Maxim,Pueraria lobata (Willd.) Ohwi, Liriope platyphylla Wang. et Tang, Panax ginseng C. A. Meyer, Poria cocos Wolf, Astragalus membranaceus Bunge, Prunus mume Siebold et Zucc., Glycyrrhiza uralensis Fisch

Odootang

Pueraria lobata (Willd.) Ohwi, Dryopteris crassirhizoma NAKAI, Glycyrrhiza uralensis Fisch, Glycine semen, Glycine max Merrill, Vigna radiata (L.) R. Wilczek., Phaseolus angularis W. F. WIGHT.

Ojeubockcheonwhan

Coptis japonica Makino, Pueraria lobata (Willd.) Ohwi, Trichosanthes kirilowii Maxim, Anemarrhena asphodeloides Bunge, Liriope platyphylla Wang. et Tang, Schizandra chinensis Bailon, Panax ginseng C. A. Meyer, Rehmannia glutinosa Libosch., Prunus mume Siebold et Zucc., Nelumbo nucifera Gaertn, Angelica gigas Nakai, Glycyrrhiza uralensis Fisch

Omeamogwatang

Prunus mume Siebold et Zucc., Chaenomeles sinensis Koehne., Hordeum vulgare L., Amomum tsao-ko Crevost et Lemarie, Glycyrrhiza uralensis Fisch

Palmisingiwhan

Rehmannia glutinosa Libosch., Dioscorea batatas Decne., Cornus officinalis S. et Z., Alisma orientale Juz., Paeonia suffruticosa Andr, Poria cocos Wolf, Schizandra chinensis Bailon, Cinnamomum cassia Blume 
Korean J. Plant Res. 25(6) : 670 681 (2012)

Table 1. Continued

\begin{tabular}{|c|c|}
\hline Herbmed & Prescription \\
\hline Saengjingamrotang & $\begin{array}{l}\text { Gypsum, Gentiana scabra Bunge, Phellodendron amurense Rupr., Bupleurum falcatum L., Ostericu } \\
\text { koreana Kitagawa, Astragalus membranaceus Bunge, Anemarrhena asphodeloides Bunge, Scutellar } \\
\text { baicalensis Georgi, Angelica gigas Nakai, Cimicifuga heracleifolia Kom., Ledebouriella seseloide } \\
\text { (Hoffm.) Wolff, Sinomenium acutum Rehder et E. H. Wilson, Rehmannia glutinosa Libosch., } \\
\text { Glycyrrhiza uralensis Fisch, Prunus armeniaca L. var. ansu Maxim,Prunus persica (L.) Batsch, } \\
\text { Carthamus tinctorius L. }\end{array}$ \\
\hline Saengjinyanghyeltang & $\begin{array}{l}\text { Angelica gigas Nakai, Paeonia japonica (Makino) Miyabe, Rehmannia glutinosa Libosch., Liriope } \\
\text { platyphylla Wang. et Tang, Cnidium officinale Makino, Coptis japonica Makino, Trichosanthes } \\
\text { kirilowii Maxim, Anemarrhena asphodeloides Bunge, Phellodendron amurense Rupr., Nelumbo } \\
\text { nucifera Gaertn, Prunus mume Siebold et Zucc., Mentha arvensis var. piperascens MALINV., } \\
\text { Glycyrrhiza uralensis } \text { Fisch }\end{array}$ \\
\hline
\end{tabular}

Samooltang

Rehmannia glutinosa Libosch., Paeonia japonica (Makino) Miyabe, Cnidium officinale Makino, Angelica gigas Nakai

Samsohwan

Sangbaekpitang

Coptis japonica Makino, Liriope platyphylla Wang. et Tang, Pueraria lobata (Willd.) Ohwi

Morus alba Linne, Poria cocos Wolf, Panax ginseng C. A. Meyer, Liriope platyphylla Wang. et Tang, Pueraria lobata (Willd.) Ohwi, Dioscorea batatas Decne., Cinnamomum cassia Blume, Glycyrrhiza uralensis Fisch

Seangjihwangeuja

Panax ginseng C. A. Meyer, Rehmannia glutinosa Libosch., Astragalus membranaceus Bunge, Asparagus cochinchinensis Merr., Liriope platyphylla Wang. et Tang, Citrus aurantium L.,

Dendrobium nobile Lindl., Eriobotrya japonica (Thunb.) Lindley, Alisma orientale Juz.

Seangjihwanggo

Rehmannia glutinosa Libosch., pis indiraRadoszkowski, Poria cocos Wolf, Panax ginseng C. A. Meyer.

Seokjajenitang

Japanese lady bell, Gypsum, Panax ginseng C. A. Meyer ,Poria cocos Wolf, Trichosanthes kirilowii Maxim, Magenetitum, Anemarrhena asphodeloides Bunge, Pueraria lobata (Willd.) Ohwi, Scutellaria baicalensis Georgi, Glycyrrhiza uralensis Fisch, Sus scrofa var. domesticus, Glycine semen

Singihwan

Rehmannia glutinosa Libosch., Dioscorea batatas Decne., Cornus officinalis S. et Z., Alisma orientale Juz., Paeonia suffruticosa Andr, Poria cocos Wolf, Schizandra chinensis Bailon

Soongisan

Magnolia officinalis Rehder et Wilson, Rheum undulatum L., Poncirus trifoliata Rafinesqul

Wesangcheonwhawon

Coptis japonica Makino, Dolichos lablab L, Aloe vera L. , Cinnabaris, Poria cocos Wolf, Ostrea gigas Thunb, Anemarrhena asphodeloides Bunge, Sophora flavescens Ait, Trichosanthes kirilowii Maxim

Whalhyulyunjosangjineum

Asparagus cochinchinensis Merr., Liriope platyphylla Wang. et Tang, Schizandra chinensis Bailon, Trichosanthes kirilowii Maxim, Cannabis sativa, Angelica gigas Nakai, Rehmannia glutinosa Libosch., Trichosanthes kirilowii Maxim, Glycyrrhiza uralensis Fisch

Whanggeumtang

Scutellaria baicalensis Georgi, Gardenia jasminoides Ellis, Platycodon grandiflorum A. DC, Liriope platyphylla Wang. et Tang, Angelica gigas Nakai, Rehmannia glutinosa Libosch., Trichosanthes kirilowii Maxim, Pueraria lobata (Willd.) Ohwi, Panax ginseng C. A. Meyer, Paeonia japonica (Makino) Miyabe, Prunus mume Siebold et Zucc.

Whanggitang

Rehmannia glutinosa Libosch., Astragalus membranaceus Bunge, Poria cocos Wolf, Trichosanthes kirilowii Maxim, Liriope platyphylla Wang. et Tang, Schizandra chinensis Bailon, Glycyrrhiza uralensis Fisch 
Table 1. Continued

\begin{tabular}{|c|c|}
\hline Herbmed & Prescription \\
\hline Whangryonjiwhangtang & $\begin{array}{l}\text { Coptis japonica Makino, Rehmannia glutinosa Libosch., Trichosanthes kirilowii Maxim, Schizandra } \\
\text { chinensis Bailon, Angelica gigas Nakai, Panax ginseng C. A. Meyer, Pueraria lobata (Willd.) Ohwi, } \\
\text { Poria cocos Wolf, Liriope platyphylla Wang. et Tang, Glycyrrhiza uralensis Fisch, Zingiber officinale } \\
\text { Roscoe, Zizyphus jujuba var. inermis,Sasa japonica Makino var. purpurascens Nakai }\end{array}$ \\
\hline Whangryunjeodoowhan & $\begin{array}{l}\text { Sus scrofa var. domesticus, Coptis japonica Makino, Triticum aestivum L., Trichosanthes kirilowii } \\
\text { Maxim, Poria cocos Wolf, Liriope platyphylla Wang. et Tang }\end{array}$ \\
\hline Whangryunjeodoowhan & $\begin{array}{l}\text { Sus scrofa var, Coptis japonica Makino, Liriope platyphylla Wang. et Tang, Anemarrhena } \\
\text { asphodeloides Bunge, Trichosanthes kirilowii Maxim }\end{array}$ \\
\hline Whanhgiyuckiltang & Astragalus membranaceus Bunge, Glycyrrhiza uralensis Fisch \\
\hline Woozeumgo & $\begin{array}{l}\text { Rehmannia glutinosa Libosch., Bos taurus coreannae, Coptis japonica Makino, Trichosanthes } \\
\text { kirilowii Maxim, Zingiber officinale Roscoe, Apis indira Radoszkowski }\end{array}$ \\
\hline Yeojigo & $\begin{array}{l}\text { Bos taurus var. domesticus Gmelin, Apis indira Radoszkowski, Prunus mume Siebold et Zucc., } \\
\text { Zingiber officinale Roscoe, Moschus moschiferus }\end{array}$ \\
\hline Yindongwon & Lonicera japonica Thunb. \\
\hline Yongbongwon & Dioscorea batatas Decne., Cuscuta chinensis Lamark, Cervus nippon hortulorum \\
\hline Yukmigeehwanghwan & $\begin{array}{l}\text { Rehmannia glutinosa Libosch., Dioscorea batatas Decne., Cornus officinalis S. et Z., Alisma orientale } \\
\text { Juz., Paeonia suffruticosa Andr, Poria cocos Wolf }\end{array}$ \\
\hline
\end{tabular}

Table 2. Selective anti-diabetes effects of $50 \% \mathrm{EtOH}$ extracts of Oriental herbs and frequency of herbs in prescription of Donguibogam

\begin{tabular}{|c|c|c|c|c|c|c|}
\hline \multirow{2}{*}{ Plant species } & \multirow{2}{*}{$\begin{array}{c}\text { Plant } \\
\text { part }\end{array}$} & \multirow{2}{*}{$\begin{array}{c}\text { Korean } \\
\text { name }\end{array}$} & \multirow{2}{*}{ Frequency } & \multicolumn{2}{|c|}{ PPAR- $\gamma$ agonist activity (\%) } & \multirow{2}{*}{$\begin{array}{c}\text { a-glucosidase } \\
\text { inhibition rate } \\
\qquad(\%)\end{array}$} \\
\hline & & & & $100 \mu \mathrm{g} / \mathrm{ml}$ & $50 \mu \mathrm{g} / \mathrm{ml}$ & \\
\hline \multicolumn{7}{|l|}{ Alismataceae } \\
\hline Alisma orientale Juz. & Rhizome & Tacksa & 4 & - & $108.41 \pm 11.12$ & - \\
\hline \multicolumn{7}{|l|}{ Amaranthaceae } \\
\hline Achyranthes bidentata Blume & Root & Wooseul & 1 & - & - & - \\
\hline \multicolumn{7}{|l|}{ Araceae } \\
\hline Pinellia ternata Ten. et Breittenb. & Tuber & Banha & 1 & - & - & - \\
\hline \multicolumn{7}{|l|}{ Araliaceae } \\
\hline Panax ginseng C. A. Meyer. & Root & Insam & 23 & - & - & 64.8 \\
\hline \multicolumn{7}{|l|}{ Aristolochiaceae } \\
\hline Asarum heterotropoides F. Maekawa & Root & Seshin & 1 & - & - & - \\
\hline \multicolumn{7}{|l|}{ Campanulaceae } \\
\hline Platycodon grandiflorum A. DC. & Root & Gilgyung & 3 & - & - & - \\
\hline \multicolumn{7}{|l|}{ Caprifoliaceae } \\
\hline Lonicera japonica Thunb. & $\begin{array}{l}\text { Leaf, } \\
\text { stem }\end{array}$ & Indong & 1 & - & - & - \\
\hline
\end{tabular}


Table 2. Continued

\begin{tabular}{|c|c|c|c|c|c|c|}
\hline \multirow{2}{*}{ Plant species } & \multirow{2}{*}{$\begin{array}{l}\text { Plant } \\
\text { part }\end{array}$} & \multirow{2}{*}{$\begin{array}{l}\text { Korean } \\
\text { name }\end{array}$} & \multirow{2}{*}{ Frequency } & \multicolumn{2}{|c|}{ PPAR- $\gamma$ agonist activity (\%) } & \multirow{2}{*}{$\begin{array}{c}\text { a-glucosidase } \\
\text { inhibition rate } \\
\qquad(\%)\end{array}$} \\
\hline & & & & $100 \mu \mathrm{g} / \mathrm{ml}$ & $50 \mu \mathrm{g} / \mathrm{ml}$ & \\
\hline \multicolumn{7}{|l|}{ Compositae } \\
\hline Atractylodes japonika Koidz. & Rhizome & Backchul & 3 & - & $44.56 \pm 11.65$ & 37.7 \\
\hline Aucklandia lappa Decne. & Root & Mockhyang & 2 & - & - & - \\
\hline Carthamus tinctorius L. & Flower & Honghwa & 3 & - & - & - \\
\hline Chrysanthemum indicum $\mathrm{L}$. & Flower & Gamgook & 1 & - & - & - \\
\hline \multicolumn{7}{|l|}{ Convolvulaceae } \\
\hline Cuscuta chinensis Lamark & Seed & Tosaja & 2 & - & - & 85.3 \\
\hline \multicolumn{7}{|l|}{ Cornaceae } \\
\hline Cornus officinalis $\mathrm{S}$. et Z. & Fruit & Sansooyou & 4 & - & - & 90.3 \\
\hline \multicolumn{7}{|l|}{ Cucurbitaceae } \\
\hline Trichosanthes kirilowii Maxim & Seed & Gwarooin & 1 & - & - & - \\
\hline Trichosanthes kirilowii Maxim & Root & Cheonhwaboon & 17 & - & - & - \\
\hline \multicolumn{7}{|l|}{ Dioscoreaceae } \\
\hline Dioscorea batatas Decne. & Rhizome & Sanyack & 7 & - & - & - \\
\hline \multicolumn{7}{|l|}{ Ephedraceae } \\
\hline Ephedra sinica & Root & Mahwangguen & 1 & $69.95 \pm 0.97$ & $98.22 \pm 9.64$ & 89.5 \\
\hline \multicolumn{7}{|l|}{ Gentianaceae } \\
\hline Gentiana scabra Bunge & Root & Yongdamcho & 1 & - & - & 15.6 \\
\hline \multicolumn{7}{|l|}{ Gramineae } \\
\hline Hordeum vulgare L. & Fruit & Meacka & 1 & - & - & - \\
\hline $\begin{array}{l}\text { Sasa japonica Makino var. } \\
\text { purpurascens Nakai }\end{array}$ & Leaf & Joockyeop & 2 & $78.69 \pm 5.79$ & $140.68 \pm 72.28$ & 67.6 \\
\hline Triticum aestivum $\mathrm{L}$. & Fruit & Boosomeack & 2 & - & - & - \\
\hline \multicolumn{7}{|l|}{ Labiatae } \\
\hline $\begin{array}{l}\text { Agastache rugosa (Fisc.r et Mey.) } \\
\text { Kuntze }\end{array}$ & Whole & Gwackhyang & 1 & $72.65 \pm 14.30$ & $76.20 \pm 7.03$ & 65.7 \\
\hline Cinnamomum cassia Blume & Cortex & Gyepea & 1 & - & - & 89.2 \\
\hline Schizonepeta tenuifolia Briq. & Spica & Hyunggea & 1 & - & - & 28.4 \\
\hline Scutellaria baicalensis Georgi & Root & Hwanggeum & 6 & - & - & - \\
\hline \multicolumn{7}{|l|}{ Leguminosae } \\
\hline Astragalus membranaceus Bunge & Root & Hwanggy & 8 & $83.58 \pm 14.91$ & $106.79 \pm 19.86$ & - \\
\hline Dolichos lablab L. & Seed & BackPyendoo & 1 & - & - & - \\
\hline Glycyrrhiza uralensis Fisch & Root & Gamcho & 32 & - & - & 62.4 \\
\hline Psoralea corylifolia L. & Seed & Pagogee & 1 & - & - & 89.8 \\
\hline Pueraria lobata (Willd.) Ohwi & Root & Galguen & 14 & - & - & 30.7 \\
\hline Sophora flavescens Ait. & Root & Gosam & 1 & - & - & 60.5 \\
\hline \multicolumn{7}{|l|}{ Liliaceae } \\
\hline Anemarrhena asphodeloides Bunge & Rhizome & Geemo & 15 & - & - & - \\
\hline Asparagus cochinchinensis Merr. & Tuber & Cheonmoondong & 3 & $17.48 \pm 8.28$ & $25.70 \pm 9.61$ & - \\
\hline Liriope platyphylla Wang. et Tang & Tuber & Makcmoondong & 20 & - & - & - \\
\hline
\end{tabular}


Table 2. Continued

\begin{tabular}{|c|c|c|c|c|c|c|}
\hline \multirow{2}{*}{ Plant species } & \multirow{2}{*}{$\begin{array}{l}\text { Plant } \\
\text { part }\end{array}$} & \multirow{2}{*}{$\begin{array}{l}\text { Korean } \\
\text { name }\end{array}$} & \multirow{2}{*}{ Frequency } & \multicolumn{2}{|c|}{ PPAR- $\gamma$ agonist activity (\%) } & \multirow{2}{*}{$\begin{array}{c}\text { a-glucosidase } \\
\text { inhibition rate } \\
\qquad(\%)\end{array}$} \\
\hline & & & & $100 \mu \mathrm{g} / \mathrm{ml}$ & $50 \mu \mathrm{g} / \mathrm{ml}$ & \\
\hline \multicolumn{7}{|l|}{ Magnoliaceae } \\
\hline Magnolia officinalis Rehder et Wilson & Cortex & Hoobark & 1 & - & - & 84.9 \\
\hline \multicolumn{7}{|l|}{ Menispermaceae } \\
\hline $\begin{array}{l}\text { Sinomenium acutum Rehder et E. H. } \\
\text { Wilson }\end{array}$ & Rhizome & Banggi & 3 & - & - & 47.1 \\
\hline \multicolumn{7}{|l|}{ Moraceae } \\
\hline Cannabis sativa & Seed & Majainn & 1 & - & - & - \\
\hline \multicolumn{7}{|l|}{ Nymphaeaceae } \\
\hline Nelumbo nucifera Gaertn. & Seed & Younja & 4 & - & - & 37.6 \\
\hline \multicolumn{7}{|l|}{ Orchidaceae } \\
\hline Dendrobium nobile Lindl. & Whole & Seockgock & 1 & - & - & 50.5 \\
\hline \multicolumn{7}{|l|}{ Orobanchaceae } \\
\hline Cistanche deserticola Y.C. Ma & Whole & Yoouckjongyong & 1 & $15.57 \pm 26.95$ & $27.65 \pm 20.00$ & 29.2 \\
\hline \multicolumn{7}{|l|}{ Ostreidae } \\
\hline Ostrea gigas Thunb. & Testa & Morea & 1 & - & - & 37.2 \\
\hline \multicolumn{7}{|l|}{ Paeoniaceae } \\
\hline Paeonia japonica (Makino) Miyabe & Root & Beackjackyack & 4 & - & - & 92.4 \\
\hline Paeonia suffruticosa Andr. & $\begin{array}{l}\text { Root } \\
\text { cortex }\end{array}$ & Mockdanpea & 4 & $14.12 \pm 21.54$ & $73.47 \pm 1.86$ & 93.2 \\
\hline \multicolumn{7}{|l|}{ Plantaginaceae } \\
\hline Plantago asiatica $\mathrm{L}$. & Seed & Chajeonja & 1 & - & - & - \\
\hline \multicolumn{7}{|l|}{ Polygalaceae } \\
\hline Polygala tenuifolia Willd. & Root & Wonjea & 1 & - & - & 87.8 \\
\hline \multicolumn{7}{|l|}{ Polygonaceae } \\
\hline Rheum undulatum L. & Rhizome & Deahwang & 3 & $17.85 \pm 60.86$ & $32.91 \pm 2.92$ & - \\
\hline \multicolumn{7}{|l|}{ Polyporaceae } \\
\hline Poria cocos Wolf & Hoelen & Backbokryuong & 16 & $90.84 \pm 1.37$ & - & - \\
\hline \multicolumn{7}{|l|}{ Ranunculaceae } \\
\hline Cimicifuga heracleifolia Kom. & Rhizome & Seungma & 4 & $21.82 \pm 8.78$ & $68.90 \pm 4.90$ & 54.7 \\
\hline Coptis japonica Makino & Rhizome & Hwangryun & 11 & $19.54 \pm 6.93$ & $24.51 \pm 16.76$ & - \\
\hline \multicolumn{7}{|l|}{ Rosaceae } \\
\hline Chaenomeles sinensis Koehne. & Fruit & Mogwa & 1 & - & - & 85.6 \\
\hline Eriobotrya japonica (Thunb.) Lindley & Leaf & Beepayeop & 1 & - & - & 81.5 \\
\hline Prunus armeniaca L. var. ansu Maxim. & Seed & Hangin & 3 & - & - & - \\
\hline Prunus mume Siebold et Zucc. & Fruit & Omea & 6 & - & - & - \\
\hline Prunus persica (L.) Batsch. & Seed & Doin & 3 & - & - & - \\
\hline \multicolumn{7}{|l|}{ Rubiaceae } \\
\hline Gardenia jasminoides Ellis & Fruit & Cheeja & 2 & - & - & 26.7 \\
\hline \multicolumn{7}{|l|}{ Rutaceae } \\
\hline Citrus aurantium $\mathrm{L}$. & Fruit & Jeegack & 3 & - & - & 44.6 \\
\hline Phellodendron amurense Rupr. & Cortex & Hwangbeack & 8 & - & - & 39.6 \\
\hline
\end{tabular}


Korean J. Plant Res. 25(6) : 670 681 (2012)

Table 2. Continued

\begin{tabular}{|c|c|c|c|c|c|c|}
\hline \multirow{2}{*}{ Plant species } & \multirow{2}{*}{$\begin{array}{c}\text { Plant } \\
\text { part }\end{array}$} & \multirow{2}{*}{$\begin{array}{l}\text { Korean } \\
\text { name }\end{array}$} & \multirow{2}{*}{ Frequency } & \multicolumn{2}{|c|}{ PPAR- $\gamma$ agonist activity (\%) } & \multirow{2}{*}{$\begin{array}{c}\text { a-glucosidase } \\
\text { - inhibition rate } \\
(\%)\end{array}$} \\
\hline & & & & $100 \mu \mathrm{g} / \mathrm{ml}$ & $50 \mu \mathrm{g} / \mathrm{ml}$ & \\
\hline Poncirus trifoliata Rafinesqul & Fruit & Jeeseal & 1 & $32.33 \pm 12.03$ & $38.47 \pm 19.07$ & 71.0 \\
\hline Zanthoxylum bungeanum & Fruit & Cheoncho & 1 & - & - & 57.0 \\
\hline \multicolumn{7}{|l|}{ Schisandraceae } \\
\hline Schizandra chinensis Bailon & Fruit & Omija & 18 & - & - & - \\
\hline \multicolumn{7}{|l|}{ Scrophulariaceae } \\
\hline Rehmannia glutinosa Libosch. & Root & Jihwang & 25 & - & - & - \\
\hline Scrophularia buergeriana Miq. & Root & Hyunsam & 2 & - & - & - \\
\hline \multicolumn{7}{|l|}{ Solanaceae } \\
\hline Lycium chinense Mill. & Cortex & Jigolpi & 3 & - & - & 21.8 \\
\hline \multicolumn{7}{|l|}{ Umbelliferae } \\
\hline Angelica gigas Nakai & Root & Dangguey & 12 & - & $12.57 \pm 6.80$ & 77.9 \\
\hline Bupleurum falcatum $\mathrm{L}$. & Root & Sihyo & 4 & - & - & 62.7 \\
\hline Cnidium officinale Makino & Rhizome & Cheongoong & 2 & $86.38 \pm 4.71$ & $113.00 \pm 5.86$ & - \\
\hline $\begin{array}{l}\text { Ledebouriella seseloides (Hoffm.) } \\
\text { Wolff }\end{array}$ & Root & Bangpoong & 2 & - & - & - \\
\hline Ostericum koreana Kitagawa & Root & Ganghwal & 2 & - & - & - \\
\hline \multicolumn{7}{|l|}{ Zingiberaceae } \\
\hline Amomum cardamomum L. & Fruit & Baeckdoogoo & 1 & - & - & - \\
\hline Amomum tsao-ko Crevost et Lemarie & Fruit & Chogwa & 1 & - & - & 65.4 \\
\hline Amomum villosum Lour. & Fruit & Sain & 1 & - & - & 81.2 \\
\hline \multicolumn{3}{|c|}{ Rosiglitazone $(10 \mu \mathrm{M})$} & & \multicolumn{2}{|c|}{$167.46 \pm 8.70$} & $\mathrm{ND}$ \\
\hline \multicolumn{3}{|l|}{ Control } & & \multicolumn{2}{|c|}{$0.0 \pm 2.59$} & $0.0 \pm 0.23$ \\
\hline
\end{tabular}

ND: Not determined, -: Inactive.

traditional prescriptions and 89 kinds of herbs in those prescriptions. These traditional medicine herbs were used in present.

\section{Herb materials and preparation}

We had investigated 71 herb plants, except for medicines that are not currently used in 89 herbs. These 71 herb plants were supplied from Semyung University Oriental Hospital in Korea. Semyung University Oriental Hospital has a clinical trial center and can study clinical trial study. Also Semyung University Oriental Hospital has treated diabetes mellitus patients and other disease by Korea oriental disease.

\section{Preparation of plant extracts}

Dried herbs were purchased and Oriental medicine doctors used the dried herbs. Herb plants part extracted for each species are shown in Table 2. Herb plants were extracted with $50 \% \mathrm{EtOH}$ for 24 hours at room temperature, after they were concentrated under reduced pressure. Samples were freeze-dried and dissolved in dimethylsulfoxide (DMSO). The final sample concentration in DMSO was $10 \mathrm{mg} / \mathrm{ml}$. Samples were kept in freezer at $-20^{\circ} \mathrm{C}$.

\section{PPAR- $\gamma$ agonist test}

3T3-L1 (mouse fibroblast) cell line was obtained from American Type Culture Collection (ATCC, USA). 3T3-L1 cell was grown in Dulbecco's modified eagle's medium (Gibco, USA) supplement with 10\% (v/v) calf serum (Invitrogen, USA) in a humidified incubator at $37^{\circ} \mathrm{C}$ and $5 \% \mathrm{CO}_{2}$. 3T3-L1 cells were seeded into 96-well plates (Nunc, USA) under the 
growth conditions described above $\left(1 \times 10^{4}\right.$ cells/well). To induce differentiation, 2-day post confluent 3T3-L1 cells (designated day 0 ) were fed DMEM containing 10\% fetal bovine serum (Gibco, USA), $1 \mu \mathrm{M}$ dexamethasone (Sigma, USA), and 0.5 mM 3-isobutyl-1-methylxanthine (Sigma, USA) until day 2. Cells then were fed DMEM supplemented with $10 \%$ FBS for 2 day, after which they were fed every other day with DMEM containing 10\% FBS. Extract was dissolved in DMSO (Sigma, USA) and was added to the PBS at indicated concentration. The cells were harvested 8 days after the initiation of differentiation for AdipoRed staining an indicator of cell differentiation rate. The cells were washed with phosphate buffered saline (PBS, pH 7.2) twice, fixed with $4 \%$ formaldehyde (Sigma, USA) at room temperature for $5 \mathrm{~h}$, and stained with AdipoRed assay reagent (Lonza, USA) for $10 \mathrm{~min}$. After 10 minutes, they were placed on the plate in the fluorometer (Perkin elmer, USA) and the fluorescence with excitation at $485 \mathrm{~nm}$ and emission $535 \mathrm{~nm}$ was measured. PPAR- $\gamma$ agonist activity (\%) was calculated using the following formula:

PPAR- $\gamma$ agonist activity $(\%)=\left(\right.$ Fluorescence test sample $^{-}$ fluorescence $_{\text {control }} / /$ fluorescence $_{\text {control }} \times 100$

Rosiglitazone (10 $\mu \mathrm{M}$ : Alexis Biochemicals, Hornby, ON), a PPAR- $\gamma$ agonist of the thiazolidinedione family, was used as a positive control, while vehicle in proliferation medium was used as a negative control.

\section{a-glucosidase inhibition assay}

Samples $(10 \mu \mathrm{l})$ were added to a 96 -well containing $0.1 \mathrm{M}$ sodium phosphate buffer $(\mathrm{pH} 6.8,84 \mu \mathrm{l})$ and $50 \mathrm{mM}$ paranitrophenyl glucopyranoside $(5 \mu \mathrm{l}), 5 \mathrm{U}$ a-glucosidase $(1 \mu \mathrm{l})$, then incubated for 20 minutes at $37^{\circ} \mathrm{C}$. The reaction was stopped by addition $200 \mu \mathrm{l}$ of $2 \mathrm{M} \mathrm{NaOH}$ and then, placed on the plate in the ELISA reader (Moledular Devices, USA). The optic density (OD) of the well at $405 \mathrm{~nm}$ was measured and the inhibition rate was calculated using following formula:

Inhibition rate $(\%)=\left(\mathrm{OD}_{\text {control }}-\mathrm{OD}_{\text {test sample }}\right) / \mathrm{OD}_{\text {control }} \times 100$

\section{Results and Discussion}

Many herb medicines have been used in the treatment of diabetes in Korea. Herb medicines have in general not associated with marked toxic or side effects. But the action mechanism of natural medicines is not clarified. Although some herbal medicines have been reported to inhibit a -glucosidase activity, most of the experiments have been limited to in vitro studies or chemical-induced diabetic animal models only (Li et al., 2005). Also PPAR- $\gamma$ agonist tests have been used to anti-diabetic effects of some herbal medicines. But these PPAR- $\gamma$ agonist tests induced the complete differentiation of 3T3-L1.

In this study, we screened herb medicines for PPAR- $\gamma$ agonist activity like TZDs (rosiglitazone). The TZD classes of insulin-sensitizing compounds such as rosiglitazone and pioglitazone, which lower hyperglycemia, are PPAR- $\gamma$ agonists (Staels and Fruchart, 2005). However, the limitations and the side-effects of these drugs have driven researchers to look for a new type of drug that could be of benefit for the treatment of some of these metabolic disorders. We used MD media (isobutyl-methylxanthine dexamethasone) to differentiate preadipocyte and total 16 herb plants had a PPAR- $\gamma$ agonist activity (Table 2). Specially, Alisma orientale Juz. (108.41\%), Ephedra sinica (98.22\%), Sasa japonica Makino var. purpurascens Nakai (140.68\%), Astragalus membranaceus Bunge (106.79\%), Cnidium officinale Makino (113.00\%) showed high PPAR- $\gamma$ agonist activity rates compared with rosiglitazone's (167.46\%).

a-Glucosidase is one of a number of glucosidase located in the brush-border surface membrane of intestinal cells, and is a key enzyme of carbohydrate digestion (Caspary, 1978). a -Glucosidase inhibitors block the actions of a-glucosidase enzymes in the small intestine, which is rate-limiting in the conversion of oligosaccharides and disaccharides to monosaccharides, necessary for gastrointestinal absorption. Postprandial glucose peaks may be attenuated by delayed glucose absorption. The main benefits attributable to a-glucosidase inhibitors are reductions in both postprandial glycemic levels and in the total range of postprandial glucose levels (Chiasson et al., 1994; Lebovitz, 1998). However, it is well documented that synthetic a-glucosidase inhibitors have undesirable side effects, such as flatulence, diarrhea and abdominal cramping. In addition, 
some of them may increase the incidence of renal tumors and serious hepatic injury and acute hepatitis (Charpentier et al., 2000; Moller, 2001).

In this a-glucosidase inhibition assay, 36 herbs had a -glucosidase inhibition (Table 2). Specially, Cornus officinalis S. et Z. (90.3\%), Cinnamomum cassia Blume (89.2\%), Psoralea corylifolia L. (89.8\%), Paeonia japonica (Makino) Miyabe (92.4\%), Paeonia suffruticosa Andr (93.2\%), showed high a -glucosidase inhibition rates.

In this study, we found out the anti-diabetic effect of herb medicine in Korean traditional medicine by PPAR- $\gamma$ agonist test and a-glucosidase inhibition assay. Our results of this study provide insight into effects and action mechanism of these herbs, a traditional Korea medicine for diabetes.

\section{Acknowledgement}

This study was supported by funding the Semyung University in 2012.

\section{Literature Cited}

Caspary, W.F. 1978. Sucrose malabsorption in man after ingestion of alpha-glucosidehydrolase inhibitor. Lancet 1:1231-1233.

Cerasi, E. 2000. Type 2 diabetes: to stimulate or not to stimulate the cell. Metabolism 49(suppl 2):1-2.

Charpentier, G., J.P. Riveline and M. Varroud-Vial. 2000. Management of drugs affecting blood glucose in diabetic patients with renal failure. Diabet. Metabol. 26:73-85.

Cheng, A. and I. Fantus. 2005. Oral antihyperglycemic therapy for type 2 diabetes mellitus. Can. Med. Assoc. J. 172:213-226. Chiasson, J.L., R.G. Josse, J.A. Hunt, C. Palmason, N.W. Rodger, S.A. Ross, E.A. Ryan, M.H. Tan and T.M. Wolever. 1994. The efficacy of acarbose in the treatment of patients with non-insulin-dependent diabetes mellitus. A multicenter controlled clinical trial. Ann. Int. Med. 15:928-935.

Evans, R.M., G.D. Barish and Y.X. Wang. 2004. PPARs and the complex journey to obesity. Nat. Med. 10:355-361.

Gerhold D.L., F. Liu, G. Jiang, Z. Li, J. Xu, M. Lu, J.R. Sachs, A. Bagchi, A. Fridman, D.J. Holder, T.W. Doebber, J. Berger, A. Elbrecht, D.E. Moller and B.B. Zhang. 2002. Gene expression profile of adipocyte differentiation and its regulation by peroxisome proliferation-activated receptor-gamma agonists.
Endocrinology 143:2106-2118.

Hansen, B.C. 1999. The metabolic X syndrome. Ann. NY Acad. Sci. 892:1-24.

Harnack, L.J., S.A. Rydell and J. Stang, 2001. Prevalence of use of herbal products by adults in the Minneapolis/St Paul, Minn, metropolitan area. Mayo. Clin. Proc. 76:688-694.

Huang, C., Y. Zhang, Z. Gong, X. Sheng, Z. Li, W. Zhang and Y. Qin. 2006. Berberine inhibits 3T3-L1 adipocyte differentiation through the PPARgamma pathway. Biochem. Biophys. Res. Commun. 348:571-578.

Kameda, K., M. Chikaki, C. Morimoto, M. Jiang and H. Okuda. 1996. Insulin like action of trans-10-hydrixy-2-decanoic acid and its related substance. J. Trad. Med. 13:456-457.

Krenisky, J.M., J. Luo, M.J. Reed and J. R. Carney. 1999. Isolation and antihyperglycemic activity of bakuchoil from Otholobium pubsecens (Fabaceae), a Peruvian medical plant used for the treatment of diabetes. Biol. Pharm. Bull. 22: 1137-1140.

Lebovitz, H.E. 1998. Postprandial hyperglycaemic state: importance and consequences. Diabetes. Res. Clin. Pract. 40:S27-S28.

Li, Y., S. Wen, B.P. Kotaa, G. Penga, G.Q. Li, J. Yamahara and B.D. Roufogalis. 2005. Punica granatum flower extract, a potent alpha-glucosidase inhibitor, improves postprandial hyperglycemia in Zucker diabetic fatty rats. J. Ethnopharmacol. 99:239-244.

Marles, R. and N. Farnsworth. 1995. Antidiabetic plants and their active constituents. Phytomedicine 2:137-165.

Matthaei, S., M. Stumvoll, M. Kellerer and H. Haring. 2000. Pathophysiology and pharmacological treatment of insulin resistance. Endocr. Rev. 21:585-618.

Moller, D.E. 2001. New drug targets for type 2 diabetes and the metabolic syndrome, Nature 414:821-827.

Pakala, R., P. Kuchulakanti, S.W. Rha, E. Cheneau, R. Baffour and R. Waksman. 2004. Peroxisome proliferator-activated receptor gamma, its role in metabolic syndrome. Cardiovasc. Radiat. Med. 5:97-103.

Stales, B. and J.C. Fruchart, 2005. Therapeutic roles of peroxisome proliferatoractivated receptor agonists. Diabetes 54:2460-2470.

Skyler, J. 2004. Diabetes mellitus: pathogenesis and treatment strategies. J. Med. Chem. 47:4113-4117.

van de Venter, M., S. Roux, L.C. Bungu, J. Louw, N.R. Crouch, O.M. Grace, V. Maharaj, P. Pillay, P. Sewnarian, N. Bhagwandin and P. Folb. 2008. Antidiabetic screening and scoring of 11 plants traditionally used in South Africa. J. Ethnopharmacol. 
119:81-86.

Wolsko, P.M., D.M. Eisenberg, R.B. Davis, S.L. Ettner and R.S. Phillips. 2002. Insurance coverage, medical conditions, and visits to alternative medicine providers: results of a national survey. Arch. Intern. Med. 162:281-287.
Yamamoto, H., S. Kurebayashi, T. Hirose, H. Kouhara and S. Kasayama. 2002. Reduced IRS-2 and GLUT4 expression in PPARgamma2-nduced adipocytes derived from C/EBP beta and C/EBP delta-deficient mouse embryonic fibroblasts. J. Cell. Sci. 115:3601-3607.

(Received 7 December 2012 ; Revised 11 December 2012 ; Accepted 12 December 2012) 